Transverse Momentum Measurement of Secondary Particles in Proton-Emulsion Collisions at $800 \mathrm{GeV}$

K. Imaeda, M. Fuki and H. Kobayashi

Department of Physics, Okayama University of Science

\title{
ABSTRACT
}

Momentum distribution of secondary particles at large angular region in proton-emulsion interactions is measured by using emulsion stack technics.

\section{THE PURPOSE OF EXPERIMENT}

It is argued that the baryon stopping power in nucleus is larger than the early estimation(1). In this regard, energies/ transverse momenta of secondary particles from proton collision with heavy nucleus are expected to be larger than proton-proton collisions. The purpose of this experiment is to measure the transverse momenta of secondary particles at large angular region from proton-emulsion $(\mathrm{Ag} / \mathrm{Br})$ interactions by scattering angle measurement in nuclear emulsion stack. Angular distribution as we11 as multiplicity distribution of p-Em interactions can also be obtained.

We propose to expose emulsion stack to $800 \mathrm{GeV}$ proton beam at Fermi laboratory.

\section{DETECTOR}

The detector is constituted of thick emulsions stacked with cross section area of $3 \times 2 \mathrm{~cm}^{2}$ and depth $5 \mathrm{~cm}$, with regard to beam direction.

\section{REQUIRED EXPERIMENTAL CONDITIONS}

1) Beam energy: mono-energetic proton beam of $800 \mathrm{GeV}$.

2) Amount of irradiation: $3 \times 10^{4} / \mathrm{cm}^{2}$ within an error of 50 percent.

\section{REFERENCE}

1) W.Busza, A.S.Goldhaber Phys. Lett. 139B(1984) 235. 